\title{
High expression of VAT1 is a prognostic biomarker and predicts malignancy in glioblastoma
}

\author{
XIA SHAN $^{1 *}$, KUANYU WANG $^{1 *}$, XUEZHI TONG ${ }^{2}$, ZHILIANG WANG $^{1}$, \\ FAN WU ${ }^{1}$, XING LIU ${ }^{1}$, PEI YANG ${ }^{2}$ and JIANGFEI WANG ${ }^{2}$ \\ ${ }^{1}$ Department of Molecular Neuropathology, Beijing Neurosurgical Institute; ${ }^{2}$ Department of Neurosurgery, \\ Beijing Tiantan Hospital, Capital Medical University, Beijing 100050, P.R. China
}

Received August 3, 2018; Accepted February 15, 2019

DOI: 10.3892/or.2019.7276

\begin{abstract}
Vesicle amine transport protein 1 (VAT1) has been reported as a pathogenic factor in a variety of tumors. VAT1 has been revealed to be upregulated in glioblastoma (GBM) and promotes cell migration. However, the possible mechanism of VAT1 in promoting malignant development in GBM is unclear. The present study applied transcriptome data and functional experiments to explore the exact role of VAT1. Kaplan-Meier survival analysis, univariate and multivariate Cox analyses were used to perform survival analysis. Furthermore, Gene Ontology analysis was used to analyze the biological implication of VAT1 expression. The in vitro experiment was performed to verify the hypothesis. The expression of VAT1 was detected in gliomas and control tissues. A functional experiment was performed and the sensitivity to TMZ was assessed after knocking down the expression of VAT1. In total, 120 patients with GBM were enrolled in the present study. The results of multivariate analysis revealed that VAT1 was an independent prognostic factor for survival. Patients with high VAT1 expression levels had shorter overall survival $(\mathrm{P}=0.009)$ and progression-free survival $(\mathrm{P}=0.055)$ than those with low expression levels. Gene Ontology analysis revealed that the genes which were positively associated with VAT1 were functionally involved in proteolysis, oxidation-reduction processes and immune response. The results of functional experiments demonstrated that VAT1 exhibited high expression levels in GBM, which could be inhibited by microRNA-218. Upon VAT1 knockdown, cell proliferation and migration were markedly suppressed, while the sensitivity toward temozolomide
\end{abstract}

Correspondence to: Dr Jiangfei Wang or Dr Pei Yang, Department of Neurosurgery, Beijing Tiantan Hospital, Capital Medical University, 6 Tiantan Xili, Dongcheng, Beijing 100050, P.R. China

E-mail: wjf1998@sohu.com

E-mail: pein19871208@163.com

${ }^{*}$ Contributed equally

Key words: VAT1, glioblastoma, prognosis, malignancy, chemotherapy chemotherapy was enhanced. Thus, VAT1 expression was revealed to be a prognostic factor for GBM. High expression of VAT1 may promote cell proliferation, migration and temozolomide chemotherapy-resistance, which may be a potential therapeutic target for GBM.

\section{Introduction}

Gliomas are primary brain tumors with high prevalence and malignancy in adults (1). Due to the high invasiveness and relapse rate, selecting a therapeutic strategy to improve the poor prognosis of malignant gliomas is a worldwide challenge $(2,3)$. For glioblastoma (GBM), the median survival time is 14.6 months despite the standard therapeutic strategy (4). The mechanisms of tumorigenesis and malignant transformation remain unknown. Previous studies have focused on aberrantly expressed or mutated genes to provide a more comprehensive understanding of tumor development and potential therapeutic targets (5-7).

Vesicle amine transport protein 1 (VAT1), a 41-kDa integral membrane protein, is embedded in synaptic vesicles and serves to transfer monoamines, including norepinephrine, epinephrine and dopamine (DA), between the cytosol and synaptic vesicles $(8,9)$. VAT1 was first described in the electric organ of the Pacific electric ray Torpedo carlifonica (10). The position of the VAT1 gene was directly adjacent to BRCA1, which was associated with inherited predisposition to breast and ovarian cancer $(6,11)$. It has been reported that VAT1 expression is not only restricted to neuronal tissues but is also present in normal epithelial tissues. The expression of VAT1 can be influenced by the extracellular calcium concentration (12-14). The association between VAT1 expression and diseases has been studied previously. VAT1 was regarded as a pathogenic factor in benign prostatic hyperplasia and was associated with cell proliferation (15). VAT1 was also regarded as a potential oncogene in gastric cancer (16). In addition, Mertsch et al observed that VAT1 was overexpressed in GBM and was functionally involved in glioma cell migration (17).

The present study aimed to detect the expression level of VAT1 in patients with GBM through RNA sequencing (RNAseq) data from public databases, and to reveal the potential relevance of VAT1 expression in tumor malignancy. Applying Gene Ontology (GO) analysis and experimental 
techniques, the present study further explored the biological function of VAT1 as a potential marker of GBM.

\section{Materials and methods}

Sample and data collection. RNAseq data of the Chinese Glioma Genome Atlas (CGGA) and The Cancer Genome Atlas (TCGA) were obtained from public available websites. From the CGGA dataset (http://www.cgga.org.cn/), 120 transcriptome data of patients diagnosed with GBM were included in the study, which were detected by the Illumina HiSeq platform (Illumina, Inc., San Diego, CA, USA) and had complete long-term follow-up information. From TCGA dataset (https://tcgadata.nci.nih.gov/tcga/tcgaDownload.jsp), the public transcriptome data of 131 patients with GBM were also acquired to serve as the validation group. Normal brain tissues and glioma tissues were obtained from surgery at Tiantan Hospital (Beijing, China). We collected 6 paired tissues and our study included 4 males and 2 females. The present study was approved by the Ethics Committee of Capital Medical University (Beijing, China). Written informed consent was obtained from all individual participants included in the study.

Cell culture. The human GBM cell line LN229 was purchased from Shanghai Bioleaf Biotech Co., Ltd. (Shanghai, China). The cells were cultured using Dulbecco's modified Eagle's medium (DMEM) containing 10\% fetal bovine serum (FBS) with $5 \% \mathrm{CO}_{2}$ and saturated humidity at $37^{\circ} \mathrm{C}$. The cells were passaged at a proportion of $1: 3$ when cell density reached $80 \%$. The 293T cell line was purchased from the Shanghai Institute of Biochemistry and Cell Biology, Chinese Academy of Sciences (Shanghai, China).

Reverse transcription-quantitative polymerase chain reaction $(R T-q P C R)$. Total RNA was isolated from cells and glioma tissues using TRIzol reagent (Aidalb Biotechnologies Co., Ltd., Beijing, China) following the manufacturer's instructions. RNA concentration was detected by NanoDrop (NanoDrop Technologies; Thermo Fisher Scientific, Inc., Wilmington, DE, USA). The RNA quality was estimated by determining the optical density (OD)260/OD280 ratio; values between 1.8 and 2.1 were considered to meet the experimental requirements. Total RNA $(1 \mu \mathrm{g})$ was transcribed into complementary DNA (cDNA) applying HiScript RT Reagent kit (Vazvme Ltd., Nanjing, China) according to the manufacturer's instructions. Upon the synthesis of cDNA, $1 \mu \mathrm{l}$ reaction volume was utilized for RT-qPCR detection on an ABI 7900 or Viia7 Fast Real-Time PCR system (Applied Biosystems; Thermo Fisher Scientific, Inc., Waltham, MA, USA). The primers used in used in VAT1 and $\beta$-actin messenger RNA detection were designed by Tsingke Biological Technology, Co., Ltd. (Beijing, China) and were as follows: VAT1 forward, 5'-TGCCGTACAGTGGAGAATGT-3' and reverse, 5'-TAGGT GACGACTTTGCCCAT-3'; and $\beta$-actin forward, 5 '-AGCGA GCATCCCCCAAAGTT-3' and reverse, 5'-GGGCACGAAG GCTCATCATT-3'. The PCR conditions were as follows: Pre-denaturation at $95^{\circ} \mathrm{C}$ for $10 \mathrm{~min}$, followed by 40 cycles of denaturation at $95^{\circ} \mathrm{C}$ for $30 \mathrm{sec}$, annealing at $60^{\circ} \mathrm{C}$ for $30 \mathrm{sec}$ and extension at $72^{\circ} \mathrm{C}$ for $30 \mathrm{sec}$. Relative gene expression was normalized to the expression of $\beta$-actin and was calculated by applying the $2^{-\triangle \Delta \mathrm{Cq}}$ method (18). The experiments were repeated $\geq 3$ times.

Cell transfection RNA interference experiments. Three types of VAT1 small interfering RNA (siRNA) were designed by 100BIOTECH, Ltd. and transfected into LN229 cells. The following VAT1 siRNA sequences were used in the present study: siRNA-1, sense GGGAGAAGUUGGGAAGCUACG and antisense, UAGCUUCC CAACUUCUC CCUU; siRNA-2, sense GGGCUUGACCAGUUCCCAAUC and antisense, UUGGGAACUGGUCAAGCCCAG; siRNA-3, sense GCU UUGGAGGCUACGACAAGG and antisense, UUGUCG UAGCCUCCAAAGCCG. The VAT1 siRNA and negative control (NC) siRNA were synthesized by 100BIOTECH, Ltd. (Hangzhou, China). The sequence of mimics NC was UUC UCCGAACGUGUCACGUTT-ACGUGACACGUUCGGAG AATT and miR-218 mimics was UUGUGCUUGAUCUAAC CAUGU-AUGGUUAGAUCAAGCACAAUU. Cells were seeded into 6-well plates (Corning Incorporated, Corning, NY, USA) at a density of $2 \times 10^{5}$ cells/well and cultured at $37^{\circ} \mathrm{C}$ with $5 \% \mathrm{CO}_{2}$ for $24 \mathrm{~h}$ before transfection. A mixture of Lipofectamine 2000 Reagent (Invitrogen; Thermo Fisher Scientific, Inc.) and siRNA was added into 6-well plates. Cells were cultured at $37^{\circ} \mathrm{C}$ with $5 \% \mathrm{CO}_{2}$ for $6 \mathrm{~h}$, and the medium was then replaced. After transfection for $48 \mathrm{~h}$, RT-qPCR was applied to verify successful overexpression or knockdown. Compared with other groups, siRNA3 exhibited a stronger interference effect and was therefore applied in the subsequent assays.

For the transfection of microRNA (miR)-218 into LN229 cells, cells were seeded in 6-well plates (Corning Incorporated) at a density of $2 \times 10^{5}$ cells/well. miR-218 mimics and negative control (NC) mimics were synthesized by Shanghai GenePharma Co., Ltd. (Shanghai, China). Lipofectamine 2000 reagent was used as a transfection agent according to the manufacturer's instructions. The expression of VAT1 mRNA was detected by RT-qPCR after the LN229 cells had been transfected for $48 \mathrm{~h}$.

Proliferation assay. Cell proliferation was evaluated with a Cell Counting Kit-8 (CCK-8) Cell Proliferation and Cytotoxicity Assay kit (Beyotime Institute of Biotechnology, Haimen, China) using 96-well plates. A total of $100 \mu 1$ DMEM with $10 \% \mathrm{FBS}$ and 5,000 cells were added to each plate in triplicate for each group. After a 48-h transfection of LN229 cells, $10 \mu \mathrm{l}$ CCK-8 reaction solution was added per well during the final $4 \mathrm{~h}$ of culture. OD values were determined at a wavelength of $450 \mathrm{~nm}$ on a microplate reader (Multiskan MK3 microplate reader; Thermo Fisher Scientific, Inc.).

Transwell assay. Cells transfected for $48 \mathrm{~h}$ were digested and resuspended in serum-free DMEM. The final concentration was $1 \times 10^{5}$ cells/100 $\mu$ l DMEM. Transwell chambers were placed in a 24-well plate containing $800 \mu \mathrm{l}$ DMEM and $10 \%$ FBS. Then, $100 \mu \mathrm{l}$ diluted cell suspension was added in the upper chamber with the basal DMEM and cultured at $37^{\circ} \mathrm{C}$ with $5 \% \mathrm{CO}_{2}$ for $5 \mathrm{~h}$. Next, the Transwell chamber was removed and the non-migrated cells on the other side of the upper chamber were gently wiped off with cotton buds. Cells were fixed in $4 \%$ paraformaldehyde solution for $10 \mathrm{~min}$, 
Table I. Clinical and molecular characteristics of 120 patients.

\begin{tabular}{|c|c|c|c|c|}
\hline Variable $(\mathrm{n}, \%)$ & $\begin{array}{c}\text { Total }(\mathrm{n}, \%) \\
120\end{array}$ & $\begin{array}{l}\text { VAT1 high expression } \\
60\end{array}$ & $\begin{array}{l}\text { VAT1 low expression } \\
60\end{array}$ & $\mathrm{P}$-value \\
\hline \multicolumn{5}{|l|}{ Age, years } \\
\hline Median (range) & $49(18-81)$ & $50.5(29-71)$ & $47.5(18-81)$ & \multirow{3}{*}{0.465} \\
\hline$\geq 49$ & 62 & 33 & 29 & \\
\hline$<49$ & 58 & 27 & 31 & \\
\hline \multicolumn{5}{|l|}{ Sex } \\
\hline Male & 74 & 41 & 33 & \multirow[t]{2}{*}{0.133} \\
\hline Female & 46 & 19 & 27 & \\
\hline \multicolumn{5}{|l|}{ 1p/19q co-deletion } \\
\hline Co-deletion & 7 & 4 & 3 & \multirow[t]{2}{*}{0.969} \\
\hline Non_codeletion & 95 & 55 & 40 & \\
\hline \multicolumn{5}{|l|}{ IDH1/2 mutation } \\
\hline Mutation & 28 & 11 & 17 & \multirow[t]{2}{*}{0.195} \\
\hline Wild-type & 92 & 49 & 43 & \\
\hline \multicolumn{5}{|c|}{ MGMT promoter methylation } \\
\hline Methylation & 51 & 27 & 24 & \multirow[t]{2}{*}{0.692} \\
\hline Unmethylation & 61 & 30 & 31 & \\
\hline \multicolumn{5}{|c|}{ TERT promoter mutation } \\
\hline Mutation & 38 & 20 & 18 & \multirow[t]{2}{*}{0.931} \\
\hline Wild-type & 58 & 30 & 28 & \\
\hline \multicolumn{5}{|l|}{ TCGA subtype } \\
\hline Proneural & 27 & 13 & 14 & \multirow[t]{4}{*}{0.031} \\
\hline Neural & 12 & 2 & 10 & \\
\hline Classical & 37 & 17 & 20 & \\
\hline Mesenchymal & 44 & 28 & 16 & \\
\hline \multicolumn{5}{|c|}{ Postoperative treatment } \\
\hline RT plus TMZ & 53 & 23 & 30 & \multirow[t]{2}{*}{0.139} \\
\hline RT only & 19 & 12 & 7 & \\
\hline
\end{tabular}

VAT1, vesicle amine transport protein 1; IDH1/2, isocitrate dehydrogenase $1 / 2$. Bold print represents $\mathrm{P}<0.05$.

followed by staining with $5 \%$ crystal violet solution for $5 \mathrm{~min}$ at room temperature and washing with phosphate-buffered saline (PBS) 3 times. Cell images were captured under an inverted microscope (IX51; Leica Microsystems GmbH, Wetzlar, Germany) in 6 randomly selected fields.

Luciferase reporter assay. Two types of recombinant plasmid were designed by 100BIOTECH,Ltd., namely pYr-VAT1-3'UTR and pYr-VAT1-3'UTR-mut. 293T cells were cultured in 12-well plates at a density of $2 \times 10^{5}$ cells/well at $37^{\circ} \mathrm{C}$ and $5 \% \mathrm{CO}_{2}$ for $24 \mathrm{~h}$. Transfection was performed using Lipofectamine 2000 reagent (Thermo Fisher Scientific, Inc.), which was mixed with $200 \mu \mathrm{l}$ nucleic acid diluents [containing NC, miR-218 mimics, 3' untranslated region (UTR) and mutant 3'UTR]. Transfected cells were cultured for $24 \mathrm{~h}$ and luciferase activity was then determined with the Dual-Luciferase Reporter Assay System (Beyotime Institute of Biotechnology) according to the manufacturer's protocol. Firefly luciferase activity was normalized to Renilla luciferase activity. Each experiment was repeated thrice.
Drug sensitivity test. LN229 cells successfully transfected with siRNA were cultured in medium containing different concentrations of temozolomide (TMZ) $(0,1,5,10$ and $25 \mu \mathrm{M}$ ) (Sigma-Aldrich; Merck KGaA, Darmstadt, Germany) for $24 \mathrm{~h}$. Next, drug sensitivity was evaluated using a CCK-8 Cell Proliferation and Cytotoxicity Assay kit. CCK-8 reaction solution was added ( $10 \mu \mathrm{l} /$ well $)$ and OD values were determined at a wavelength of $450 \mathrm{~nm}$ on a microplate reader (Multiskan MK3 microplate reader) after $2 \mathrm{~h}$ of culture.

Western blot analysis. Normal brain tissues and glioma tissues were cut into pieces of $3 \times 3 \mathrm{~mm}$ and digested in RIPA Lysis Buffer (Beyotime Institute of Biotechnology). Total protein was extracted and quantified with the BCA Protein Assay kit (Beyotime Institute of Biotechnology) according to the manufacturer's protocol. Equal quantities of total protein $(40 \mu \mathrm{g})$ from the cell lysates were subjected to $10 \%$ SDS-PAGE to separate the proteins and then transferred to a polyvinylidene fluoride (PVDF) membrane (EMD Millipore, Billerica, MA, USA). After blocking in TBS-Tween-20 (TBST) (Amresco, 
Inc., Framingham, MA, USA) containing 5\% non-fat milk at room temperature for $1 \mathrm{~h}$, the membrane was incubated with a primary antibody against GAPDH (dilution 1:5,000; cat. no. AB-P-R 001; Hangzhou Goodhere Biotechnology Co., Ltd., Hangzhou, China) and VAT1 (dilution 1:800; cat. no. 22016-1-AP; ProteinTech Group, Inc., Wuhan, China) at $4^{\circ} \mathrm{C}$ overnight and washed with TBST 5-6 times (5 min each). Then, the membrane was incubated with a goat anti-rabbit immunoglobulin $\mathrm{G}$ secondary antibody conjugated to horseradish peroxidase (dilution 1:5,000; cat. no. BA1054; Boster Biological Technology Technology, Pleasanton, CA, USA), followed by immersion in enhanced chemiluminescence substrate solution (Thermo Fisher Scientific, Inc.) to detect the signals. GAPDH served as an internal control.

The LN229 cells transfected with NC or miR-218 mimics were also subjected to western blotting. Cells were washed with cold PBS and lysed in RIPA Lysis buffer (Beyotime Institute of Biotechnology). The methods of protein extraction and subsequent detection were the same as aforementioned.

Statistical analysis. Data were divided into two groups according to the median expression level of VAT1 and the statistical difference between each group was assessed by Student's t test using R and SPSS software. We used one-way analysis of variance (ANOVA) followed by Holm-Sidak test for comparisons among multiple groups. Overall survival (OS) was determined from the time of surgery to death or the last follow-up. Progression-free survival(PFS) was defined as the time from surgery to tumor recurrence or last follow-up. Multivariate analysis was performed to evaluate the influence factor of survival by the Cox regression model. Kaplan-Meier survival curves were used to estimate the survival rate. $\mathrm{P}<0.05$ was considered to indicate a statistically significant difference.

\section{Results}

VATl is an independent risk factor for patients with GBM. The clinicopathological characteristics of 120 patients with GBM from the CGGA dataset were included in our study. Their follow-up data were complete and available. Patients were divided into two groups according to the RNAseq expression level of VAT1, and each group contained 60 patients. The results of Chi-square revealed that only the TCGA subtype had a statistically significant difference between the two groups $(\mathrm{P}=0.031$; Table I). Patients in the VAT1 high-expression group tended to be of the mesenchymal subtype, while in the low-expression group, the neural subtype was more common than other types. The median age of all patients was 49 years (range, 18-81 years), and the median age of the VAT1 high-expression group was older than that of the low-expression group. However, there was no statistically significant difference in VAT1 expression levels regarding age or sex. Considering the importance of pathological factors in diagnosis and prognosis, several biomarkers that have been widely reported in the field of glioma were selected. The status of these markers exhibited no differences between the VAT1 high-expression group and the low-expression group, including 1p/19q co-deletion, isocitrate dehydrogenase $1 / 2$ (IDH1/2) mutation, MGMT promoter methylation and TERT promoter mutation. In the subsequent multivariate
Table II. Multivariate factors of the overall survival of patients.

$95 \%$ Confidence interval

\begin{tabular}{lcccc} 
Variables & P-value & Hazards ratio & Lower & Upper \\
\hline Age, years & 0.701 & 1.170 & 0.525 & 2.607 \\
Sex & 0.056 & 0.540 & 0.287 & 1.016 \\
TCGA subtype & 0.976 & 1.005 & 0.728 & 1.387 \\
VAT1 & $\mathbf{0 . 0 2 1}$ & 2.065 & 1.116 & 3.822 \\
IDH1/2 & 0.346 & 0.712 & 0.351 & 1.444 \\
1p/19q & 0.094 & 0.953 & 0.276 & 3.291 \\
MGMT & 0.905 & 1.036 & 0.584 & 1.837 \\
TERT & 0.151 & 0.631 & 0.336 & 1.184 \\
Radiotherapy & 0.083 & 0.620 & 0.361 & 1.064 \\
Chemotherapy & $\mathbf{0 . 0 2 8}$ & 0.517 & 0.287 & 0.933 \\
\hline
\end{tabular}

VAT1, vesicle amine transport protein 1; IDH1/2, isocitrate dehydrogenase $1 / 2$. Bold print represents $\mathrm{P}<0.05$.

Table III. Multivariate factors of the progression-free survival of patients.

95\% Confidence interval

\begin{tabular}{lcccc} 
Variables & P-value & Hazards ratio & Lower & Upper \\
\hline Age, years & 0.967 & 1.016 & 0.465 & 2.220 \\
Sex & 0.101 & 0.606 & 0.333 & 1.103 \\
TCGA subtype & 0.596 & 1.089 & 0.794 & 1.493 \\
VAT1 & 0.129 & 1.576 & 0.875 & 2.839 \\
IDH1/2 & 0.436 & 0.763 & 0.387 & 1.506 \\
1p/19q & 0.727 & 1.242 & 0.368 & 4.193 \\
MGMT & 0.789 & 1.076 & 0.628 & 1.846 \\
TERT & 0.723 & 0.900 & 0.503 & 1.610 \\
Radiotherapy & $\mathbf{0 . 0 4 1}$ & 0.576 & 0.338 & 0.979 \\
Chemotherapy & 0.237 & 0.703 & 0.392 & 1.261
\end{tabular}

VAT1, vesicle amine transport protein 1; IDH1/2, isocitrate dehydrogenase $1 / 2$. Bold print represents $\mathrm{P}<0.05$.

analysis, the age of patients, VAT1 expression levels and the aforementioned vital markers were taken into consideration, and the influence of each of these variables on survival was assessed. The results revealed that VAT1 was an independent risk factor for $\mathrm{OS}[\mathrm{P}=0.021$; hazard ratio $(\mathrm{HR})=2.065$; 95\% confidence interval $(\mathrm{CI})=1.116-3.822$; Table II) but not PFS ( $\mathrm{P}=0.129 ; \mathrm{HR}=1.576 ; 95 \% \mathrm{CI}=0.875-2.839$; Table III). The strategy of postoperative adjuvant treatment, which combined radiotherapy with or without TMZ chemotherapy, had no statistically significant differences between the two VAT1 expression groups.

Influence of different VAT1 expression levels on survival. Based on the results of multivariate analysis, VAT1 was identified as an independent risk factor for survival, which 
A

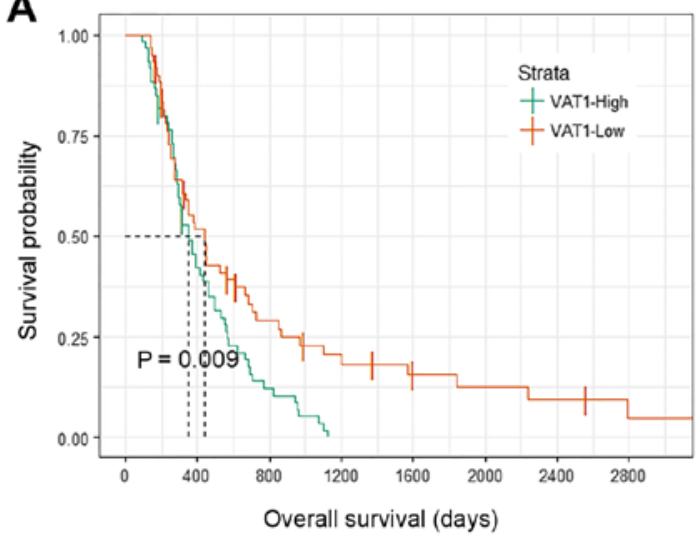

B

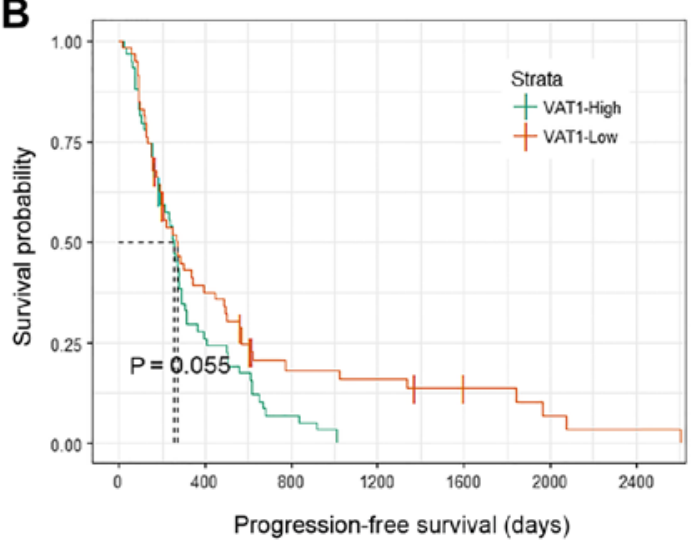

Figure 1. VAT1 is a poor prognosis factor in patients with glioblastoma. Survival analysis revealed that high expression of VAT1 indicated a shorter (A) overall survival $(\mathrm{P}=0.009)$ and $(\mathrm{B})$ progression-free survival $(\mathrm{P}=0.055)$. VAT1, vesicle amine transport protein 1 .

A

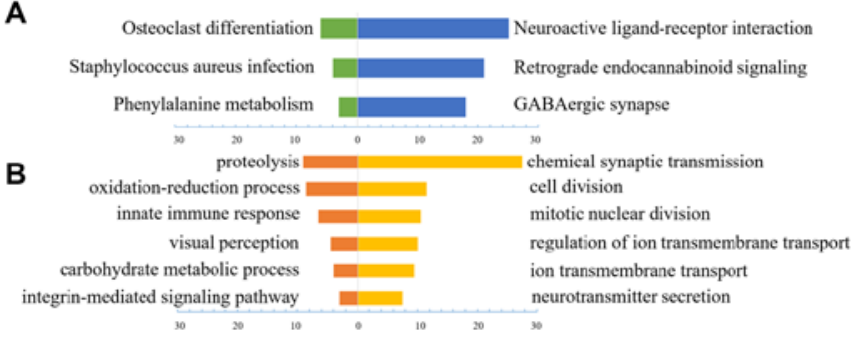

C

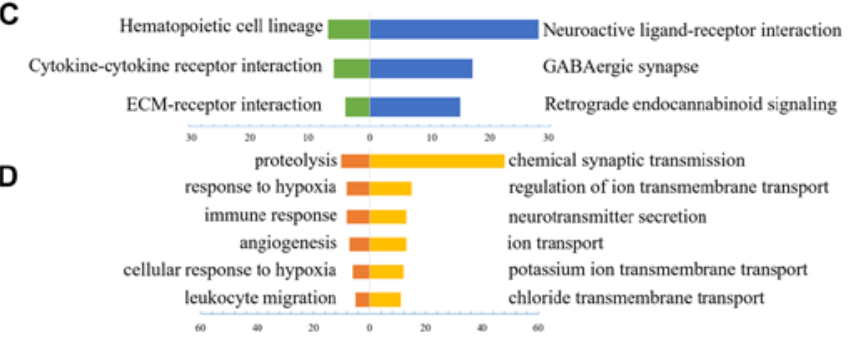

Figure 2. Gene Ontology analysis of the CGGA and TCGA datasets. (A) Pathway enrichment of differentially expressed genes in the CGGA dataset. (B) Biological function enrichment of differentially expressed genes in the CGGA dataset. (C) Pathway enrichment of differentially expressed genes in TCGA dataset. (D) Biological function enrichment of differentially expressed genes in TCGA dataset. Green and red bars represent high VAT1 expression. Blue and yellow bars represent low VAT1 expression. CGGA, Chinese Glioma Genome Atlas; TCGA, The Cancer Genome Atlas; VAT1, vesicle amine transport protein 1.

could impact the prognosis of patients. To visualize this trend, Kaplan-Meier curves were generated to demonstrate the influence of VAT1 expression on OS and PFS in our data (Fig. 1). Patients with high VAT1 expression levels had shorter OS and PFS compared with patients in the low-expression group, although only OS exhibited a statistically significant difference $(\mathrm{P}=0.009$; Fig. 1A). Combined with the results from the multivariate analysis, VAT1 may serve an important role in the development of glioma and may be associated with tumor progression and poor prognosis. Thus, the present study focused on the association between VAT1 expression and the features of patients with GBM, and further explored the function of VAT1.

VAT1-associated biological processes and signaling pathways. To investigate the biological features of patients with GBM with different VAT1 expression levels, a paired t-test was applied to evaluate the differentially expressed genes $(\mathrm{P}<0.05)$. In total, 1,038 and 776 genes in the CGGA and TCGA datasets, respectively, were available for subsequent analysis. GO analysis online with DAVID (https://david.ncifcrf.gov/) was applied to reveal the function of VAT1-associated genes. Finally, various biological processes and signaling pathways that contained a relatively low P-value and a high number of genes were selected. In general, functionally similar results for VAT1 low expression-associated genes were identified in the CGGA (Fig. 2A and B) and TCGA datasets (Fig. 2C and D). The majority of VAT1 low-expression-associated genes' biological processes were involved in chemical synaptic transmission, ion transport and neurotransmitter secretion, which may indicate various normal biological functions. The signaling pathways associated with high VAT1 expression levels in both datasets could nearly reach uniformity, while the results of biological processes analysis revealed that the function of these genes was mainly associated with proteolysis, oxidation-reduction processes and immune response. Particularly, in TCGA dataset, the genes associated with high expression levels of VAT1 were functionally involved in angiogenesis and hypoxia, which are regarded as the hallmarks of tumor development, and suggests that VAT1 may be a potential gene influencing tumorigenesis and tumor progression.

VAT1 expression levels in gliomas. The aforementioned results demonstrated the potential function of VAT1 in malignant gliomas. To further explore the role of VAT1, the present study compared the mRNA expression levels of VAT1 between gliomas and control tissues (i.e. the peritumoral tissue of glioma, which was generally located $1 \mathrm{~cm}$ away from the tumor). The results revealed that VAT1 mRNA expression was markedly higher in gliomas than in control tissues (Fig. 3B). The subsequent western blot analysis confirmed that the protein levels of VAT1 were distinctly higher in gliomas compared with peritumoral tissues (Fig. 3A). As reported in previous studies, numerous miRs appear to be 
A

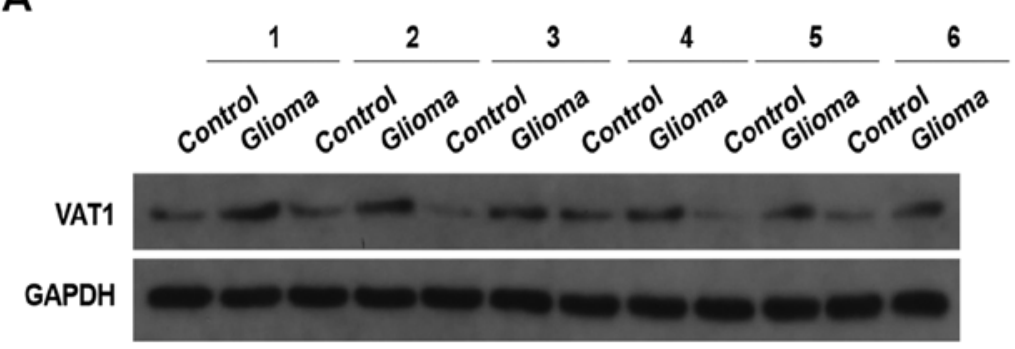

B

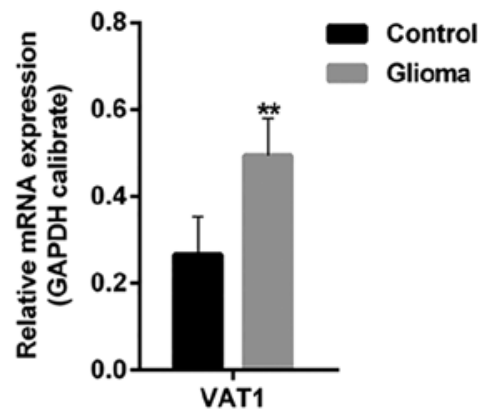

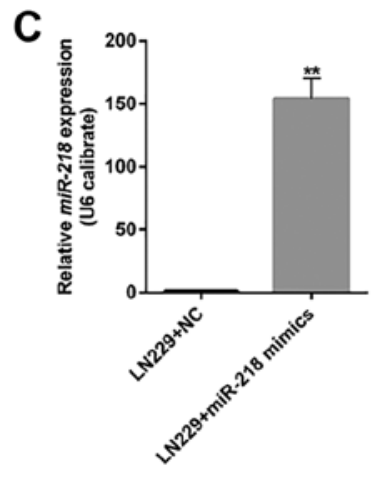
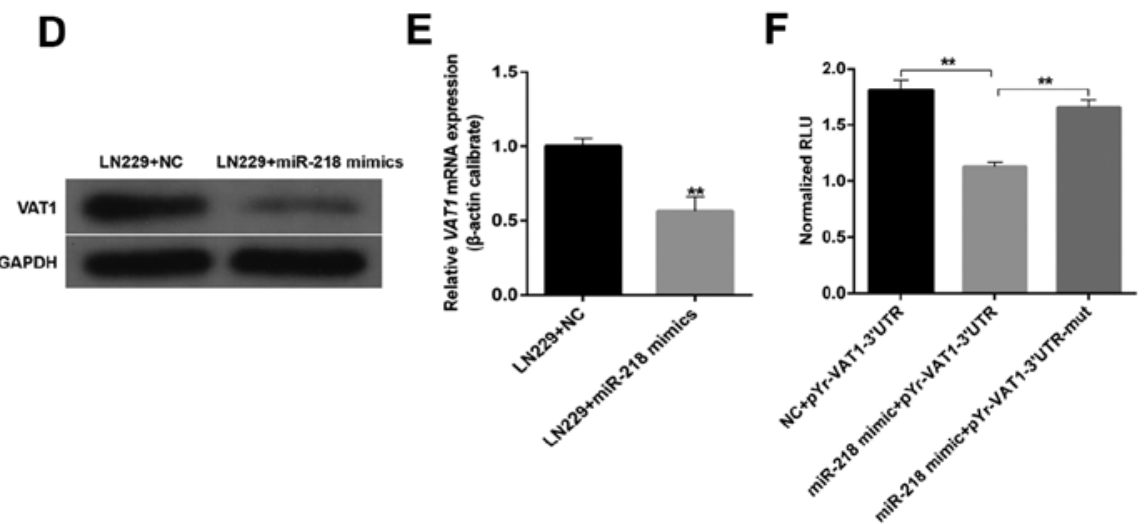

Figure 3. Detection of VAT1 expression level. (A and B) VAT1 was overexpressed in glioblastoma at the mRNA and protein level. (C-E) The mRNA and protein expression levels of VAT1 were markedly reduced upon transfection with miR-218 mimics. (F) Luciferase reporter assay demonstrated that miR-218 suppressed the expression of VAT1 in a targeted manner. VAT1, vesicle amine transport protein $1 .{ }^{* *} \mathrm{P}<0.01$.

associated with tumor initiation and progression by influencing the expression of specific genes $(18,19)$. An increasing body of evidence suggests that miR-218 may act as a tumor suppressor by decreasing cell proliferation, invasion and migration $(20,21)$. Therefore, the present study next assessed the influence of miR-218 on VAT1 expression and revealed that miR-218 inhibited the expression of VAT1 both at the mRNA and protein level. Upon transfection with miR-218 mimics, the cells exhibited markedly low expression levels of VAT1 (Fig. 3C-E). According to the results of a dual-luciferase reporter assay, compared with the NC-pYr-VAT1-3'UTR group, the relative fluorescence units (RLUs) of cells transfected with miR-218 mimic-pYr-VAT1-3'UTR were remarkably decreased (Fig. 3F), while the RLUs of cells transfected with miR-218 mimic-pYr-VAT1-3'UTR-mut were significantly increased compared with the miR-218 mimic-pYr-VAT1-3'UTR group, which suggested that miR-218 mimics played a targeted inhibitory role on VAT1.

VAT1 knockdown inhibits cell proliferation and migration, and enhances TMZ sensitivity. The direct function of VAT1 was evaluated by knocking down the gene (Fig. 4A). The results demonstrated that cell viability and the number of migrated cells were markedly decreased (Fig. 4D and E), which indicated that VAT1 could promote cell proliferation and migration. A marked decrease in cell migration was also observed upon knockdown of VAT1 (Fig. 4B-C). To evaluate the effect of VAT1 on TMZ, the present study conducted a drug sensitivity test, and the half maximal inhibitory concentration $\left(\mathrm{IC}_{50}\right)$ value was used as a quantitative indicator. The results revealed that the $\mathrm{IC}_{50}$ was decreased in the VAT1 siRNA group $\left(\mathrm{C}_{50}=3.500\right.$; Fig. $\left.5 \mathrm{~A}\right)$ compared with the $\mathrm{NC}$ group $\left(\mathrm{C}_{50}=5.108\right.$; Fig. $\left.5 \mathrm{~B}\right)$. Regarding the detection of cell inhibition rates, silencing VAT1 could improve the inhibition ratio at the same concentration of TMZ compared with the control (Fig. 5C). Thus, VAT1 may reduce the sensitivity of TMZ.

\section{Discussion}

With high malignancy and invasion features, gliomas are regarded as the most common type of brain tumor in adults. Tumor recurrence is almost inevitable despite advancements in treatment strategy (1). To further understand the characteristics of GBM and to identify novel therapeutic targets, numerous studies have focused on genes associated with tumorigenesis, invasion and prognosis. In the present study, high expression levels of VAT1 were detected in brain tissues of patients with GBM compared with normal brain tissues. Multivariate analysis revealed that VAT1 was an independent prognostic factor for OS. Kaplan-Meier curves demonstrated that high expression levels of VAT1 indicated poor prognosis. Based on these findings, it was proposed that VAT1 may serve an important role in the tumorigenesis of gliomas. However, to the best of our knowledge, only Mertcsh et al have reported to date the effect of VAT1 on glioma cell migration. Thus, the role of VAT1 in glioma remains unclear to a great extent (17). To further explore the function of VAT1, GO analysis was 

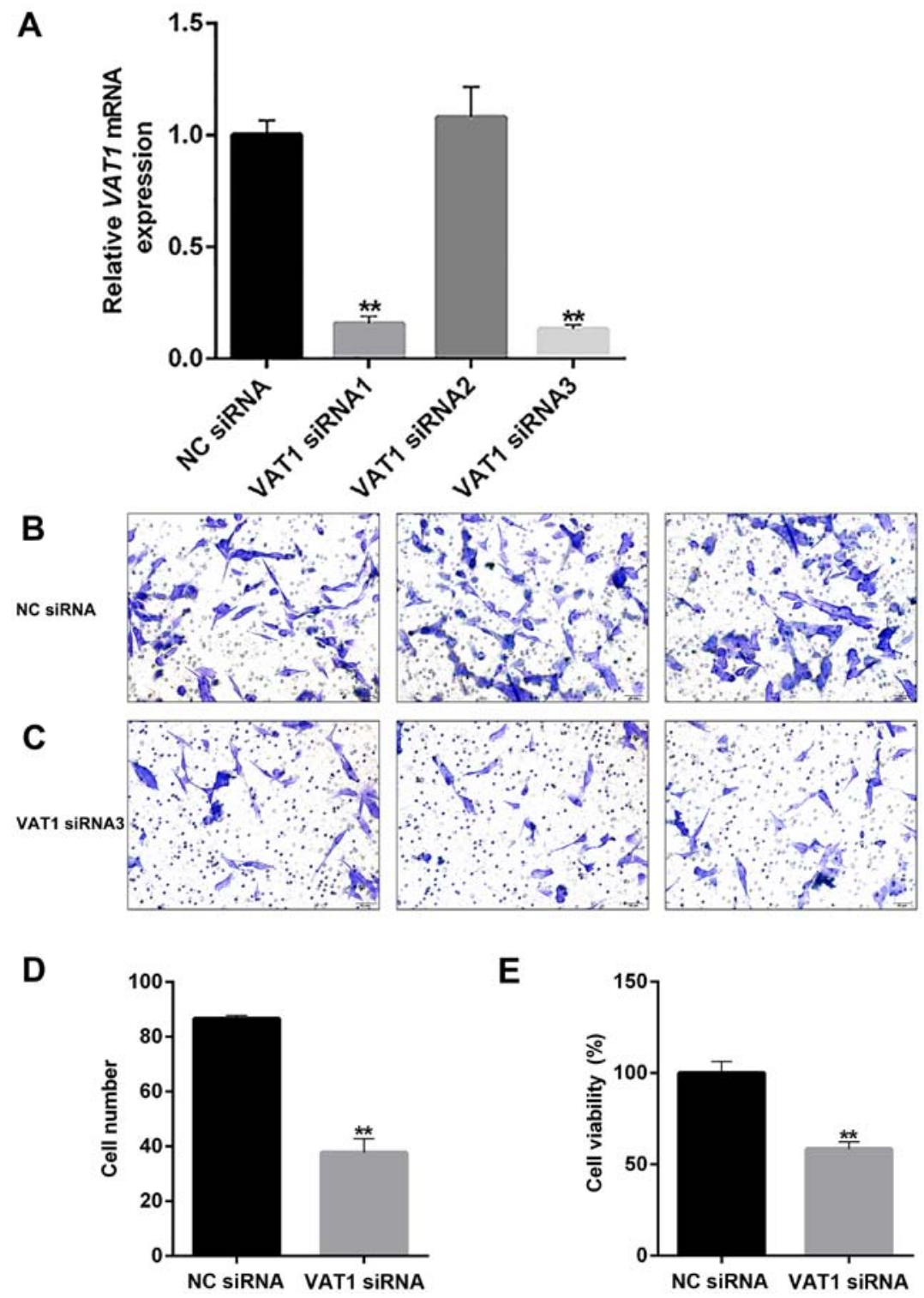

Figure 4. Functional experiments of VAT1. (A-C) Cell migration was inhibited by knocking down VAT1. In the top row, cells were transfected with negative control siRNA. In the bottom row, cells were transfected with VAT1 siRNA. The experiments were repeated thrice. (D) The number of cells was significantly decreased upon transfection with VAT1 siRNA, indicating suppressed cell migration. (E) Upon knockdown of VAT1, cell viability decreased, indicating inhibition of cell proliferation. VAT1, vesicle amine transport protein $1 .{ }^{* *} \mathrm{P}<0.01$.

A

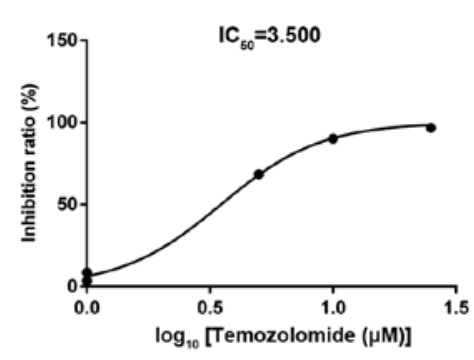

B

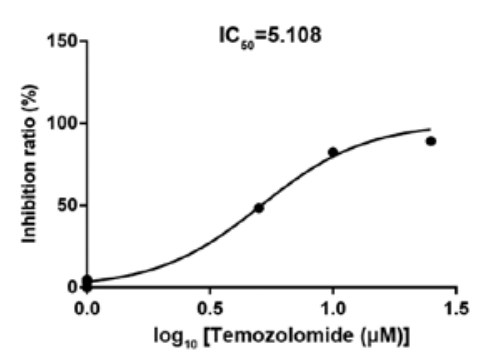

C

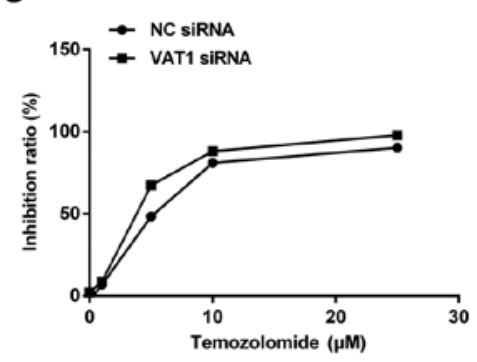

Figure 5. (A) The $\mathrm{IC}_{50}$ of TMZ was $3.500 \mu \mathrm{M}$ in the VAT1 siRNA-transfected group. (B) The $\mathrm{IC}_{50}$ of $\mathrm{TMZ}$ was $5.108 \mu \mathrm{M}$ in the negative control group. (C) The comparison of $\mathrm{IC}_{50}$ values revealed an enhanced sensitivity towards TMZ in the VAT1 siRNA-transfected group. TMZ, temozolomide; VAT1, vesicle amine transport protein 1 .

applied to reveal the relevant biological processes and pathways of differentially expressed genes based on the mRNA expression levels of VAT1. As anticipated, the major biological processes of VAT1 were chemical synaptic transmission, ion transport and neurotransmitter secretion, which was in agreement with previous research. The biological processes of 
VAT1 were positively associated with genes that were mainly involved in proteolysis, immune response, response to hypoxia and oxidation-reduction processes, which suggested that high VAT1 expression may be a positive regulatory factor for tumor proliferation and growth. VAT1 may act as a biomarker in glioma progression and exhibits potential as a novel target for the treatment of GBM.

The present results demonstrated that VAT1 expression levels were markedly high in brain tissues of patients with GBM compared with normal brain tissues. miRs (also called miRNAs) are small non-coding RNAs of $\sim 20-23$ nucleotides that regulate gene expression and various biological processes $(22,23)$. Previous studies indicated that miR-218 was markedly suppressed in tumors, and that the upregulation of miR-218 could inhibit cell proliferation and migration by targeting diverse genes (24-26). Therefore, VAT1 expression levels were detected in GBM cell lines transfected with miR-218 to assess the influence of miR-218 on VAT1. The results suggested that VAT1 expression was suppressed by miR-218. Subsequently, a series of functional experiments were performed to validate the function of VAT1. siRNA-mediated VAT1 knockdown significantly suppressed the proliferation and migration of GBM cells. Furthermore, enhancement of TMZ sensitivity was observed in VAT1-knockdown tumor cells. Thus, downregulation of VAT1 may be a potential therapeutic target in the treatment of GBM based on our results. However, further studies are required to identify the potential mechanisms by which VAT1 regulates glioma biological functions.

Notably, the function of genes positively associated with high VAT1 expression was linked with immune response. Similar results were obtained in TCGA dataset, which suggested a potential link between VAT1 expression and tumor immunity. Recently, several studies have focused on tumor-associated immunosuppression. Diverse mechanisms of escaping tumor-specific T cell-mediated immune response have been proposed by various studies (27-31). Supper et al identified a significant interaction between cluster of differentiation (CD)147 and VAT1, and regarded VAT1 as a novel interacting partner that dynamically interacts with CD147 upon T-cell activation (32). Additionally, GO analysis revealed that the neurotransmitter secretion function in genes was correlated with low VAT1 expression levels. The function of the integral membrane protein encoded by VAT1 is to transfer monoamines (e.g. dopamine). Dopamine (DA) as a vital neurotransmitter, participates in many processes in the central nervous system (CNS) and its function in the immune system has also been discussed recently. One effect of DA is the regulation of leukocyte activation and function during the immune response (33). The dopaminergic system (DAS) has a plasma membrane-specific DA transporter called sodium-dependent DA transporter. The VAT1 protein was reported to be a transporter of DAS, which was preferentially expressed in neuroendocrine cells $(34,35)$. In a previous in vitro experiment, the results revealed that incubation of $\mathrm{CD}^{+} \mathrm{CD} 25^{+}$regulatory $\mathrm{T}$ cells (Treg) with VAT1 inhibitor (e.g., reserpine) led to the downregulation of intracellular catecholamine concentrations and the increase of the concentration of the medium (36). Based on these studies, it was hypothesized that upregulation of VAT1 expression may inhibit the unleashing of DA. Furthermore, $T$ cells have the ability to synthesize and metabolize DA, and they can respond to DA by releasing and recapturing these molecules $(37,38)$. Our future studies may further focus on the interaction between VAT1 and various specific immune cells present in the tumor microenvironment.

In conclusion, GBM displays significantly high VAT1 expression levels, which indicates a more invasive, malignant status and poor prognosis. Such phenomenon may be attributable to the influence of VAT1 expression on tumor immunity. VAT1 may act as a potential therapeutic target, and its association with immune cells must be explored in future studies.

\section{Acknowledgements}

Not applicable.

\section{Funding}

The present study was supported by the Beijing Natural Science Foundation Project (grant no. 7152052) and the Beijing Tiantan Hospital Miaopu Project (grant no. 2017MP05).

\section{Availability of data and materials}

All data generated or analyzed during the present study are included in this article.

\section{Authors' contributions}

$\mathrm{XS}$ and $\mathrm{KW}$ conceived and designed the experiments and wrote the manuscript. XS, KW, XT and ZW performed the experiments and analyzed the data. FW and LX guided the experiments. XT, ZW, FW and LX helped with interpretation of data. PY and JW performed the surgery and designed the study. All authors read and approved the manuscript and agree to be accountable for all aspects of the research in ensuring that the accuracy or integrity of any part of the work are appropriately investigated and resolved.

\section{Ethics approval and consent to participate}

The present study was approved by the Ethics Committee of Capital Medical University (Beijing, China). Written informed consent was obtained from all individual participants included in the study.

\section{Patient consent for publication}

Not applicable.

\section{Competing interests}

All authors declare that they have no conflicts of interest.

\section{References}

1. Van Meir EG, Hadjipanayis CG, Norden AD, Shu HK, Wen PY and Olson JJ: Exciting new advances in neuro-oncology: The avenue to a cure for malignant glioma. CA Cancer J Clin 60: 166-193, 2010. 
2. Louis DN, Ohgaki H, Wiestler OD, Cavenee WK, Burger PC, Jouvet A, Scheithauer BW and Kleihues P: The 2007 WHO classification of tumours of the central nervous system. Acta Neuropathol 114: 97-109, 2007

3. Claes A, Idema AJ and Wesseling P: Diffuse glioma growth: A guerilla war. Acta Neuropathol 114: 443-458, 2007.

4. Stupp R, Mason WP, van den Bent MJ, Weller M, Fisher B, Taphoorn MJ, Belanger $\mathrm{K}$, Brandes AA, Marosi C Bogdahn U, et al; European Organisation for Research and Treatment of Cancer Brain Tumor and Radiotherapy Groups; National Cancer Institute of Canada Clinical Trials Group: Radiotherapy plus concomitant and adjuvant temozolomide for glioblastoma. N Engl J Med 352: 987-996, 2005.

5. Parsons DW, Jones S, Zhang X, Lin JC, Leary RJ, Angenendt P, Mankoo P, Carter H, Siu IM, Gallia GL, et al: An integrated genomic analysis of human glioblastoma multiforme. Science 321: $1807-1812,2008$.

6. Smith TM, Lee MK, Szabo CI, Jerome N, McEuen M, Taylor M, Hood L and King MC: Complete genomic sequence and analysis of $117 \mathrm{~kb}$ of human DNA containing the gene BRCA1. Genome Res 6: 1029-1049, 1996.

7. Wang Y and Jiang T: Understanding high grade glioma: Molecular mechanism, therapy and comprehensive management. Cancer Lett 331: 139-146, 2013.

8. Eiden LE, Schäfer MK, Weihe E and Schütz B: The vesicular amine transporter family (SLC18): Amine/proton antiporters required for vesicular accumulation and regulated exocytotic secretion of monoamines and acetylcholine. Pflugers Arch 447: 636-640, 2004

9. Linial M and Levius O: VAT-1 from Torpedo is a membranous homologue of zeta crystallin. FEBS Lett 315: 91-94, 1993.

10. Linial M, Miller K and Scheller RH: VAT-1: An abundant membrane protein from Torpedo cholinergic synaptic vesicles. Neuron 2: 1265-1273, 1989

11. Miki Y, Swensen J, Shattuck-Eidens D, Futreal PA, Harshman K, Tavtigian S, Liu Q, Cochran C, Bennett LM, Ding W, et al: A strong candidate for the breast and ovarian cancer susceptibility gene BRCA1. Science 266: 66-71, 1994.

12. Koch J, Foekens J, Timmermans M, Fink W, Wirzbach A Kramer MD and Schaefer BM: Human VAT-1: A calcium-regulated activation marker of human epithelial cells. Arch Dermatol Res 295: 203-210, 2003.

13. Vicanová J, Boelsma E, Mommaas AM, Kempenaar JA, Forslind B, Pallon J, Egelrud T, Koerten HK and Ponec M: Normalization of epidermal calcium distribution profile in reconstructed human epidermis is related to improvement of terminal differentiation and stratum corneum barrier formation. J Invest Dermatol 111: 97-106, 1998.

14. Owens DW, Brunton VG, Parkinson EK and Frame MC: E-cadherin at the cell periphery is a determinant of keratinocyte differentiation in vitro. Biochem Biophys Res Commun 269: 369-376, 2000

15. Mori F, Tanigawa K, Endo K, Minamiguchi K, Abe M, Yamada S and Miyoshi K: VAT-1 is a novel pathogenic factor of progressive benign prostatic hyperplasia. Prostate 71: 1579-1586, 2011.

16. Mottaghi-Dastjerdi N, Soltany-Rezaee-Rad M, Sepehrizadeh Z, Roshandel G, Ebrahimifard F and Setayesh N: Gene expression profiling revealed overexpression of vesicle amine transport protein-1 (VAT-1) as a potential oncogene in gastric cancer. Indian J Biotechnol 15: 161-165, 2016.

17. Mertsch S, Becker M, Lichota A, Paulus W and Senner V: Vesicle amine transport protein-1 (VAT-1) is upregulated in glioblastomas and promotes migration. Neuropathol Appl Neurobiol 35: 342-352, 2009.

18. Novakova J, Slaby O, Vyzula R and Michalek J: MicroRNA involvement in glioblastoma pathogenesis. Biochem Biophys Res Commun 386: 1-5, 2009.

19. Palumbo S, Miracco C, Pirtoli L and Comincini S: Emerging roles of microRNA in modulating cell-death processes in malignant glioma. J Cell Physiol 229: 277-286, 2014.
20. Gao X and Jin W: The emerging role of tumor-suppressive microRNA-218 in targeting glioblastoma stemness. Cancer Lett 353: 25-31, 2014

21. Yang Y, Ding L, Hu Q, Xia J, Sun J, Wang X, Xiong H, Gurbani D, Li L, Liu Y, et al: MicroRNA-218 functions as a tumor suppressor in lung cancer by targeting IL-6/STAT3 and negatively correlates with poor prognosis. Mol Cancer 16: 141, 2017.

22. Winter J, Jung S, Keller S, Gregory RI and Diederichs S: Many roads to maturity: microRNA biogenesis pathways and their regulation. Nat Cell Biol 11: 228-234, 2009.

23. Hwang HW and Mendell JT: MicroRNAs in cell proliferation, cell death, and tumorigenesis. Br J Cancer 94: 776-780, 2006.

24. Yu J, Wang Y, Dong R, Huang X, Ding S and Qiu H: Circulating microRNA-218 was reduced in cervical cancer and correlated with tumor invasion. J Cancer Res Clin Oncol 138: 671-674, 2012.

25. Nishikawa R, Goto Y, Sakamoto S, Chiyomaru T, Enokida H, Kojima S, Kinoshita T, Yamamoto N, Nakagawa M, Naya Y, et al: Tumor-suppressive microRNA-218 inhibits cancer cell migration and invasion via targeting of LASP1 in prostate cancer. Cancer Sci 105: 802-811, 2014.

26. Gu J, Xu R, Li Y,Zhang J and Wang S: MicroRNA-218 modulates activities of glioma cells by targeting HMGB1. Am J Transl Res 8: 3780-3790, 2016.

27. Pardoll DM: The blockade of immune checkpoints in cancer immunotherapy. Nat Rev Cancer 12: 252-264, 2012.

28. Fecci PE, Ochiai H, Mitchell DA, Grossi PM, Sweeney AE, Archer GE, Cummings T, Allison JP, Bigner DD and Sampson JH: Systemic CTLA-4 blockade ameliorates glioma-induced changes to the $\mathrm{CD}^{+} \mathrm{T}$ cell compartment without affecting regulatory T-cell function. Clin Cancer Res 13: 2158-2167, 2007.

29. See AP, Parker JJ and Waziri A: The role of regulatory T cells and microglia in glioblastoma-associated immunosuppression. J Neurooncol 123: 405-412, 2015.

30. Shukla SA, Rooney MS, Rajasagi M, Tiao G,Dixon PM,Lawrence MS, Stevens J, Lane WJ, Dellagatta JL, Steelman S, et al: Comprehensive analysis of cancer-associated somatic mutations in class I HLA genes. Nat Biotechnol 33: 1152-1158, 2015.

31. Doucette T, Rao G, Rao A, Shen L, Aldape K, Wei J, Dziurzynski K, Gilbert $M$ and Heimberger AB: Immune heterogeneity of glioblastoma subtypes: Extrapolation from the cancer genome atlas. Cancer Immunol Res 1: 112-122, 2013.

32. Supper V, Hartl I, Boulègue C, Ohradanova-Repic A and Stockinger H: Dynamic interaction- and phospho-proteomics reveal Lck as a major signaling hub of CD147 in T cells. J Immunol 198: 2468-2478, 2017.

33. Arreola R, Alvarez-Herrera S, Pérez-Sánchez G, Becerril-Villanueva E, Cruz-Fuentes C, Flores-Gutierrez EO, Garcés-Alvarez ME, de la Cruz-Aguilera DL, Medina-Rivero E, Hurtado-Alvarado G, et al: Immunomodulatory effects mediated by dopamine. J Immunol Res 2016: 3160486, 2016.

34. Essand M, Vikman S, Grawé J, Gedda L, Hellberg C, Oberg K, Totterman TH and Giandomenico V: Identification and characterization of a novel splicing variant of vesicular monoamine transporter 1. J Mol Endocrinol 35: 489-501, 2005.

35. Wimalasena K: Vesicular monoamine transporters: Structurefunction, pharmacology, and medicinal chemistry. Med Res Rev 31: 483-519, 2011.

36. Cosentino M, Fietta AM, Ferrari M, Rasini E, Bombelli R, Carcano E, Saporiti F, Meloni F, Marino F and Lecchini S: Human $\mathrm{CD} 4{ }^{+} \mathrm{CD} 25^{+}$regulatory $\mathrm{T}$ cells selectively express tyrosine hydroxylase and contain endogenous catecholamines subserving an autocrine/paracrine inhibitory functional loop. Blood 109: 632-642, 2007.

37. Amenta F, El-Assouad D, Mignini F, Ricci A and Tayebati SK: Neurotransmitter receptor expression by peripheral mononuclear cells: Possible marker of neuronal damage by exposure to radiations. Cell Mol Biol (Noisy-le-grand) 48: 415-421, 2002.

38. Musso NR, Brenci S, Setti M, Indiveri F and Lotti G: Catecholamine content and in vitro catecholamine synthesis in peripheral human lymphocytes. J Clin Endocrinol Metab 81: 3553-3557, 1996. 